\title{
Dosimetric Feasibility of Utilizing the ViewRay Magnetic Resonance Guided Linac System for Image-guided Spine Stereotactic Body Radiation Therapy
}

Gage Redler ${ }^{1}$, Tynan Stevens ${ }^{2}$, Jochen Cammin ${ }^{3}$, Martha Malin ${ }^{4}$, Olga Green ${ }^{3}$, Sasa Mutic ${ }^{3}$, Sean Pitroda ${ }^{5}$, Bulent Aydogan ${ }^{5}$

1. Radiation Oncology, Moffitt Cancer Center, Tampa, USA 2. Medical Physics, Dalhousie University, Halifax, CAN 3. Radiation Oncology, Washington University School of Medicine, Barnes-Jewish Hospital, St. Louis, USA 4. Radiation Oncology, New York University, Langone Medical Center \& Laura and Issac Perlmutter Cancer Center, New York, USA 5. Radiation and Cellular Oncology, University of Chicago, Chicago, USA

Corresponding author: Gage Redler, gage@uchicago.edu

\section{Abstract}

Introduction: Spine stereotactic body radiation therapy (SBRT) achieves favorable outcomes compared to conventional radiotherapy doses/fractionation. The spinal cord is the principal dose-limiting organ-at-risk (OAR), and safe treatment requires precise immobilization/localization. Therefore, image guidance is paramount to successful spine SBRT. Conventional X-ray imaging and alignment to surrogate bony anatomy may be inadequate, whereas magnetic resonance imaging (MRI) directly visualizes the dose-limiting cord. This work assessed the dosimetric capability of the ViewRay (ViewRay Inc. Oakwood Village, OH) magnetic resonance (MR) guided linac (MR-Linac) for spine SBRT.

Methods: Eight spine SBRT patients without orthopedic hardware who were previously treated on a TrueBeam using volumetric modulated arc therapy (VMAT) were re-planned using MR-Linac fixed-field intensity-modulated radiation therapy (IMRT). Phantom measurements using film, ionization chamber, and a commercial diode-array assessed feasibility. Plans included a variety of prescriptions (30-50 Gy in 3-10 fractions).

Results: MR-Linac plans satisfied all clinical goals. Compared to VMAT plans, both entrance dose and heterogeneity increased ( $D_{\max }: 134 \pm 3 \%$ vs. $120 \pm 2 \%$, $p=0.0270$ ), while conformality decreased (conformity index: $1.28 \pm 0.06$ vs. $1.06 \pm 0.06, p=0.0005$ ), and heterogeneity increased. However, while not statistically significant, MR-linac cord sparing improved (cord $\mathrm{D}_{\max }$ : $16.1 \pm 2.7 \mathrm{~Gy}$ vs. $19.5 \pm 1.6 \mathrm{~Gy}, \mathrm{p}=0.2066$; cord planning organ at risk volume (cord PRV) $\mathrm{D}_{\max }: 20.0 \pm 2.6 \mathrm{~Gy}$ vs. $24.5 \pm 2.0 \mathrm{~Gy}, \mathrm{p}=0.0996$ ). Delivery time increased but was acceptable (14.39 $\pm 1.26 \mathrm{~min}$ vs. $9.57 \pm 1.19 \mathrm{~min})$. Ionization chamber measurements agreed with planned dose to within $2.5 \%$. Film and diode measurements demonstrated accurate/precise delivery of dose gradients

Received 11/30/2019

Review began 12/02/2019 Review ended 12/11/2019 Published 12/12/2019

\section{() Copyright 2019}

Redler et al. This is an open access article distributed under the terms of the Creative Commons Attribution License CC-BY 3.0., which permits unrestricted use, distribution, and reproduction in any medium, provided the original author and source are credited. between the target and the cord.

Conclusion: Spine SBRT with the MR-Linac is feasible as verified via re-planning eight clinical cases followed by delivery verification in phantoms using film, diodes, and an ionization chamber. Real-time visualization of the dose-limiting cord during spine SBRT may enable cord-based gating, reduced margins, alternate dose schemas, and/or adaptive therapy.

\section{Categories: Medical Physics, Radiation Oncology}

Keywords: spine sbrt, igrt, mr-igrt, magnetic resonance-guided radiation therapy (mrgrt), dosimetry treatment planning

\section{Introduction}

Nearly $40 \%$ of cancer patients present with clinically symptomatic spinal disease and $\sim 90 \%$ demonstrate evidence of disease upon autopsy [1]. Treatment options include systemic therapy, surgery, radiation therapy (RT), or surgery+RT, with goals of pain palliation, bone fracture avoidance, and/or management of neurological symptoms and disease progression [2]. In many settings, surgery+RT is standard of care. Ablative stereotactic body RT (SBRT) may provide improved efficacy compared to conventional fractionation [1]. Furthermore, spine SBRT has improved potential for retreatment and dose escalation for radioresistant disease.

Accuracy and precision are paramount to spine SBRT, thereby emphasizing patient immobilization and image guidance. The primary dose-limiting organ-at-risk (OAR) is the spinal cord. Cord definition is usually from magnetic resonance imaging (MRI) (or computed tomography (CT) myelography). Cord delineation uncertainty, inability to visualize/immobilize the cord during treatment, and the importance of respecting 
cord tolerances often lead to a cord planning organ-at-risk volume (PRV). Use of PRV margin, typically 1-2 $\mathrm{mm}$, decreases disease coverage [1]. Prior to image-guided RT (IGRT), a framed approach provided immobilization for linac-based spine SBRT [3]. Initial IGRT attempts used a vacuum bag immobilization device and ultrasound [4]. Real-time image guidance (orthogonal kVs) with Cyberknife enabled frameless spine SBRT by providing setup uncertainties of 1.0-1.2 mm [5-6]. Similar approaches are now widely adopted using traditional linac approaches and $\mathrm{kV}$ image guidance (2D planar images and/or 3D cone beam CT (CBCT)).

An issue with conventional $\mathrm{kV}$ imaging is that positioning tends to rely on bony anatomy, which is a surrogate for the anatomy of interest (e.g., tumor or cord). MRI can address this and already has important roles in RT, specifically spine SBRT [1-2]. Factors limiting utilization of MRI in RT (e.g., logistics of having a dedicated MRI, geometric distortion, availability during treatment), have mostly been addressed with the advent of MR guided RT (MRgRT) [7-8]. One such implementation is the MRidian MR-linac (ViewRay Incorporated, Oakwood Village, OH) [7]. The utility of MRidian images has been compared to CT and onboard CBCT. No significant volumetric differences were found between contours drawn using CT versus ViewRay MRI [9]. Compared to on-board CBCT, MRIdian images were mostly superior, with the major shortcoming being bony anatomy visualization, which is less pertinent when soft tissue (e.g., cord) is directly identifiable [10].

The MRIdian MR-linac system (6MV flattening filter free) has improved dosimetric qualities compared to a prior cobalt-based system (e.g., higher multileaf collimator (MLC) resolution, improved penetration, decreased penumbra, faster treatment) [11-14]. Specifically, the MR-linac system's MLCs have a width of 8.3 $\mathrm{mm}$ but are double-stacked and offset to provide a $4.15 \mathrm{~mm}$ effective leaf width, versus $10.5 \mathrm{~mm}$ for the cobalt system. MLCs for both systems are double-focused (see [15] for details on system technical specifications). One planning study with the ${ }^{60} \mathrm{Co}$ MRIdian suggested that spine SBRT was not dosimetrically feasible [13]. MRgRT spine SBRT may be feasible with the MR-linac system, and may improve upon kV-based IGRT approaches. Preliminary data suggests that aligning to vertebra versus cord, which is visible with MRIdian, requires an average shift of $0.9 \mathrm{~mm}$ (up to $1.8 \mathrm{~mm}$ observed; no obvious time trends) [16].

This work demonstrates the dosimetric feasibility of spine SBRT with the ViewRay MR-Linac system, which may enable visualization of the dose limiting spinal cord during treatment and allow cord-based gated delivery and/or online adaptive spine SBRT. Eight spine SBRT plans (clinically planned using VMAT on a conventional linac) are compared to MR-Linac plans. Fidelity between planned and delivered MR-linac dose distributions is verified using phantom measurements with film, diodes, and ion chambers.

\section{Materials And Methods}

\section{Clinical cases}

Eight spine SBRT plans recently treated in our clinic were re-planned in the ViewRay treatment planning system (TPS). Plans included prescription doses ranging from 30 to $50 \mathrm{~Gy}$ and fractionation schemes ranging between three and ten fractions. One plan incorporated a simultaneous integrated boost. PTV sizes ranged from 25-160 cc. PTV extent ranged from anterior vertebral body only, to various combinations of pedicle, transverse process and spinous process coverage (see Figure 1 for delineation of regions of potential disease extent; see Table 1 for corresponding descriptions of targets used in this work), based upon the international spine radiosurgery consortium target volume guidelines [2]. 


\section{Cureus}

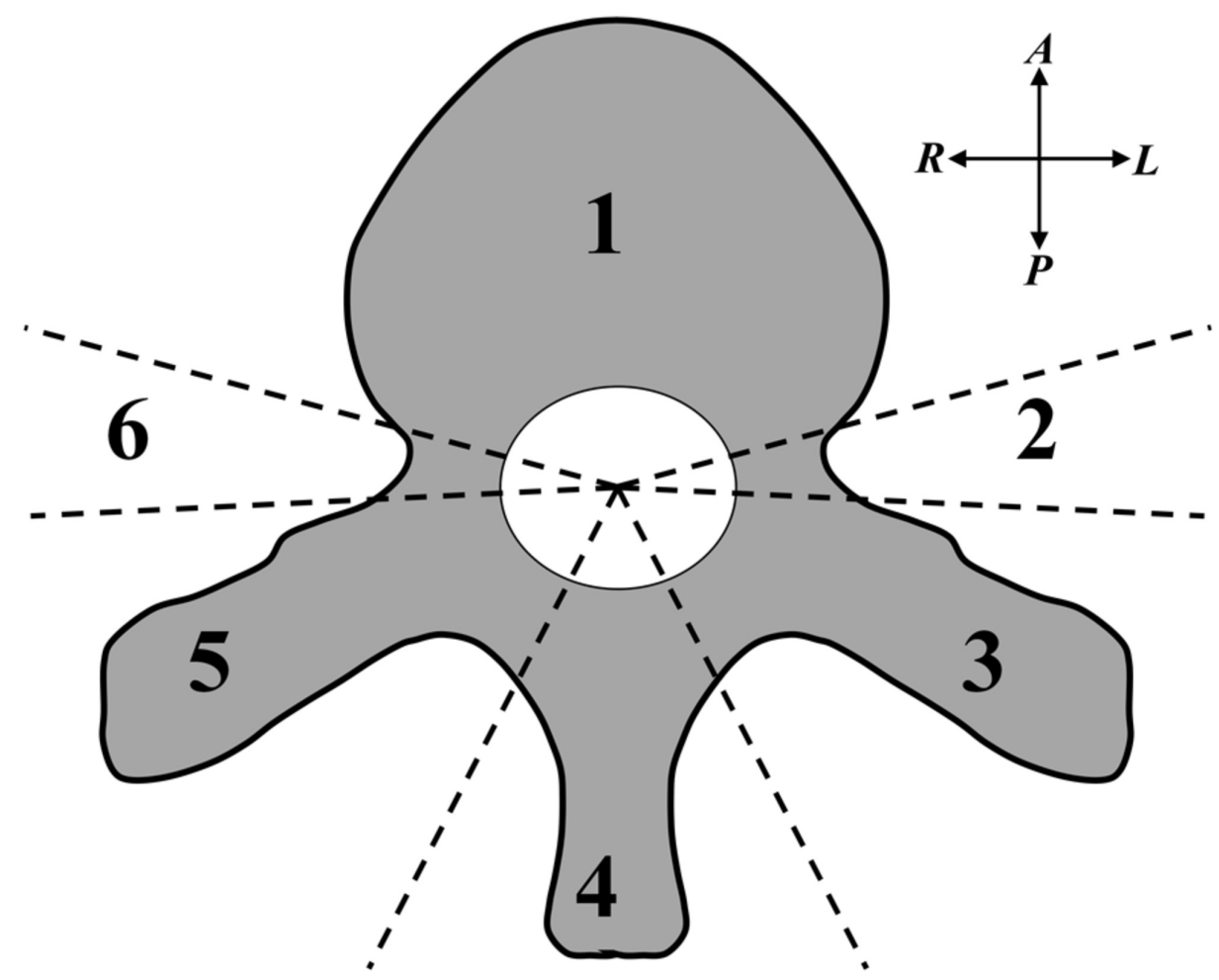

\section{FIGURE 1: Spinal Vertebrae Diagram}

Diagram of spinal vertebrae (axial plane) showing different regions by which to describe the extent of the planning target volume (PTV) to be treated. $1=$ vertebral body, $2=$ left pedicle, $3=$ left transverse process, 4 $=$ spinous process, $5=$ right transverse process, $6=$ right pedicle. The spinal canal containing the spinal cord is shown as the central white oval area.

\begin{tabular}{lllll}
\hline Case \# (Vertebral Level) & PTV Volume [cc] & PTV Extent & Prescription Dose / Fractionation & PTV Clinical Coverage Goal(s) \\
\hline 1 (T) & 60.2 & $1,2,6$ & $30 \mathrm{~Gy} / 3 \mathrm{fx}$ & $\mathrm{V}_{100 \%}>95 \%$ \\
2 (T) & 119.9 & $1,2,3,5,6$ & $50 \mathrm{~Gy} / 5 \mathrm{fx}$ & $\mathrm{V}_{100 \%}>80 \% ; \mathrm{V}_{90 \%}>95 \%$ \\
3 (T) & 38.2 & $1,2,4,5,6$ & $30 \mathrm{~Gy} / 3 \mathrm{fx}$ & $\mathrm{V}_{100 \%}>95 \%$ \\
4 (L/S) & $160.3(9.1)$ & $1,2,4,5,6$ & $30(40) \mathrm{Gy} / 5 \mathrm{fx}$ & $\mathrm{V}_{100 \%}>95 \%$ \\
5 (T) & 36.3 & $1,2,3,4,6$ & $30 \mathrm{~Gy} / 3 \mathrm{fx}$ & $\mathrm{V}_{100 \%}>90 \% ; \mathrm{V}_{90 \%}>98 \%$ \\
6 (L) & 73.7 & $1,2,6$ & $30 \mathrm{~Gy} / 3 \mathrm{fx}$ & $\mathrm{V}_{100 \%}>90 \% ; \mathrm{V}_{90} \%>98 \%$ \\
7 (L) & 25.8 & 1 & $30 \mathrm{~Gy} / 3 \mathrm{fx}$ & $\mathrm{V}_{100 \%}>95 \%$ \\
8 (T) & 71.2 & $1,2,4,5,6$ & $30 \mathrm{~Gy} / 3 \mathrm{fx}$ & $\mathrm{V}_{100 \%}>90 \%$
\end{tabular}

\section{TABLE 1: Plan Characteristics}

Description of treatment plans included in this study. Plan \#4 included a simultaneous integrated boost, which is indicated by the additional volume and $\mathrm{Rx}$ dose in parenthesis.

\section{Planning approach}

The principal dose-limiting OARs were the spinal cord and esophagus. Cord OAR definition was either based on CT myelogram $(n=6)$ or substituted with spinal canal $(n=2)$. Cord planning organ at risk volume (PRV) used a $1 \mathrm{~mm}$ expansion. When spinal canal was a cord surrogate, no PRV expansion was used. Some plans were treating regions in the lumbar/sacral vertebral level, where cord transitions to cauda equina, however, 
for generality, this distinction is not made (i.e., cauda = cord). Secondary OARs include esophagus, bowel, liver, and lungs. These were not dose-limiting and are not presented in the following analyses. OAR goals were from the American Association of Physicists in Medicine (AAPM) TG-101 [17]. All images and structures (targets, OARs, PRVs) were consistent between clinical VMAT plans and MR-Linac plans.

Conventional linac plans were VMAT and used a Varian TrueBeam accelerator with HDMLC and a 6MV beam. The plans used 2-4 co-planar arcs. PTV coverage goals were $\mathrm{V}_{100 \%}>95 \%$ unless OAR constraint(s) precluded this, in which case either fractionation was reconsidered or lower PTV coverage was accepted. Eclipse v11 (n=7) (Varian Medical Systems, Inc., Palo Alto, CA) or Pinnacle v9 (n=1) (Philips Radiation Oncology Systems, Fitchburg, WI) were used. Dose was calculated with $1 \mathrm{~mm}$ resolution, using homogeneity correction (collapsed cone convolution superposition in Pinnacle and Acuros in Eclipse).

Fixed-field IMRT was adopted for the MR-Linac plans, with nine equally-spaced co-planar beams, starting with a gantry angle of $0^{\circ}$. Due to limited space within the MR-Linac bore, the VMAT isocenter could not always be replicated. For these cases, isocenter was as close to the middle of the treated vertebral body as possible. Plans were calculated using $1 \mathrm{~mm}$ resolution, similar to conventional linac plans, using the ViewRay TPS advanced implementation (enabling dose volume histogram (DVH)-based planning constraints) and Monte Carlo dose computation algorithm (1\% dose uncertainty).

\section{Plan evaluation}

Dosimetric endpoints comparing MR-Linac and conventional linac plans included both PTV and OAR considerations. For the PTV, these were target coverage $\left(V_{100 \%}, V_{95 \%}\right.$, and $\left.V_{90 \%}\right)$, maximum dose $\left(D_{\max }\right)$ for heterogeneity, and conformity index $\left(\mathrm{CI}=\mathrm{V}_{\mathrm{Rx}} / \mathrm{V}_{\mathrm{PTV}}\right.$, where $\mathrm{V}_{\mathrm{Rx}}$ is the prescription isodose volume and $\mathrm{V}_{\mathrm{PTV}}$ is PTV volume). For the OARs/PRVs, these consisted of $\mathrm{D}_{\max }$ and organ specific dose-volume thresholds (e.g., spinal cord $\mathrm{V}_{12.3 G y}$ and $\mathrm{V}_{18 G \mathrm{y}}$, esophagus $\mathrm{V}_{17.7 \mathrm{~Gy}}$, bowel $\mathrm{V}_{17.4 G y}$, liver $\mathrm{V}_{30 \mathrm{~Gy}}$, and lung $\mathrm{V}_{20 \mathrm{~Gy}}$ ). TPS calculated beam-on time was also evaluated. Statistical significance was determined using a two-tailed paired-samples t-test.

\section{Phantom measurements}

To evaluate deliverability/accuracy of the plans and TPS calculated dose distributions, various dosimetric measurements were made for two of the eight cases, representing two ends of the spectrum of PTV geometric complexity. These cases were \#7, with the PTV solely including the vertebral body (i.e., a simple target) and \#8 with the PTV fully wrapped around the spinal cord (i.e., the most challenging target). Dosimeters were radiochromic film, a commercial quality assurance (QA) diode array, and an ionization chamber.

EBT3 Gafchromic film (Ashland Specialty Ingredients, NJ) provided a high-resolution comparison between planned and delivered dose. The accuracy of film for measurements in relatively high magnetic field environments has been established [18-19]. Film sheets $\left(10 \times 10 \mathrm{~cm}^{2}\right)$ were placed at the center of a $30 \times 30 \times 30 \mathrm{~cm}^{3}$ water-equivalent plastic phantom (Solid Water, Gammex, Middleton, WI). Film was exposed when oriented in axial, sagittal, and coronal planes. To avoid saturation, QA plans were scaled so that maximum dose was $<1000 \mathrm{cGy}$. Treatment fields were re-cast onto a CT of this phantom and the calculated planar dose corresponding to film location/orientation was used for comparison. The RIT software package (Radiological Imaging Technology, Colorado Springs, CO) was used for dosimetric comparison via global gamma analysis with $3 \% / 3 \mathrm{~mm}$ and $2 \% / 2 \mathrm{~mm}$ criteria (10\% low-dose threshold). Planned dose distribution was resampled to the higher film resolution (scanned using red channel, 150 dots per inch (DPI)).

The plans were also measured using the commercially-available, MR-compatible ArcCHECK phantom (Sun Nuclear Corporation, Melbourne, FL) with an Accredited Dosimetry Calibration Laboratory (ADCL) calibrated ionization chamber (Exradin A18MRI, Standard Imaging, Middleton, WI, collecting volume 0.123 cc) placed in the central insert. The ArcCHECK (consisting of a helical diode array) with ion chamber represents a common approach to routine patient-specific QA with the MR-Linac [19-20]. Previous studies have demonstrated accuracy of using this ion chamber in the presence of the $0.35 \mathrm{~T}$ magnetic field [19-21]. Treatment fields were re-cast onto the cylindrical ArcCHECK phantom in the TPS and the SunNuclear QA software used the resulting RTdose DICOM file to extract the planned dose plane corresponding to the cylindrical surface within the phantom containing the diode array. Comparison between planned and measured dose surfaces was performed using the same global gamma criteria $(3 \% / 3 \mathrm{~mm}$ and $2 \% / 2 \mathrm{~mm}$ criteria, with a $10 \%$ low-dose threshold).

\section{Results}

\section{Planning comparison}

A representative case comparing the clinical VMAT and MR-linac plans is in Figure 2. The MR-linac plans spare the cord well but are less conformal (see isodose lines bowing out compared to VMAT plan, particularly near PTV distal to cord). The fixed-field IMRT approach for MR-linac planning exhibits more appreciable 


\section{Cureus}

low-dose along beam directions.

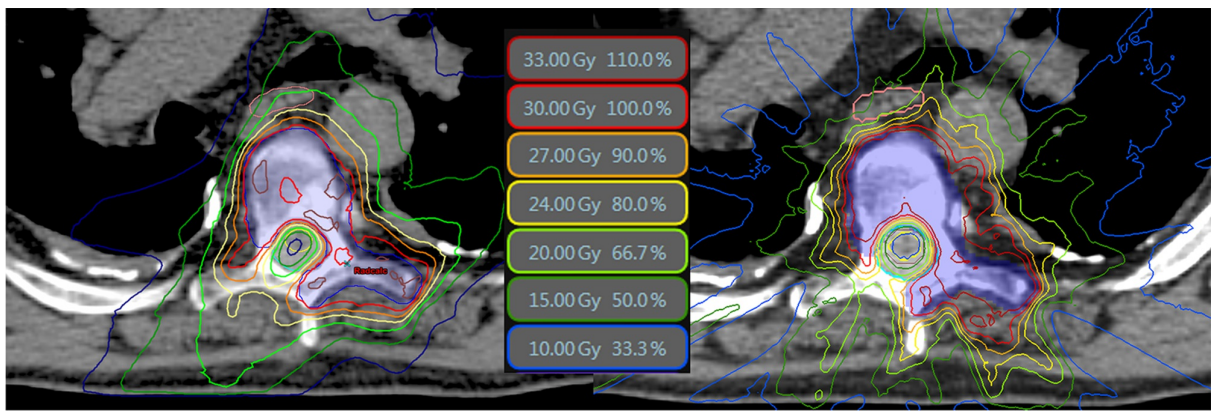

\section{FIGURE 2: Qualitative Dosimetric Comparison}

Qualitative dosimetric comparison of isodose distribution for case \#5 clinical volumetric modulated arc therapy (VMAT) plan (left) and corresponding magnetic resonance guided linac (MR-Linac) plan (right). The planning target volume (PTV) is the blue shaded region.

A comprehensive quantitative comparison of overall trends for all cases considered is in Figure 3. All MRlinac plans are clinically acceptable by our institutional spine SBRT protocol. There are no significant differences in target coverage (average PTV V 100 of MR-linac vs. clinical VMAT are $92.7 \%$ [range: 85.995.9\%] vs. $92.3 \%$ [range: $81.2-98.95 \%$ ], respectively, $\mathrm{p}=0.7572$ ). The higher conformity index demonstrates significantly inferior conformity for the MR-linac vs. VMAT plans ( $1.28 \pm 0.06$ vs. $1.06 \pm 0.06$, respectively, $\mathrm{p}=0.0005)$. Increased $\mathrm{D}_{\max }$ shows significantly increased heterogeneity for MR-linac vs. VMAT plans

$(134 \pm 3 \%$ vs. $120 \pm 2 \%$, respectively, $\mathrm{p}=0.0270$ ). While not statistically significant, MR-linac plans show trends of lower cord doses compared to VMAT plans (cord $\mathrm{D}_{\max }$ of $16.1 \pm 2.7 \mathrm{~Gy}$ vs. $19.5 \pm 1.6 \mathrm{~Gy}, \mathrm{p}=0.2066$, and cord PRV D $D_{\max }$ of $20.0 \pm 2.5$ Gy vs. $24.5 \pm 2.0 \mathrm{~Gy}, \mathrm{p}=0.0996$, for MR-linac vs. VMAT). Volumetric planning goals for the cord, cord PRV, spinal canal, and esophagus were comparable with no significant differences.
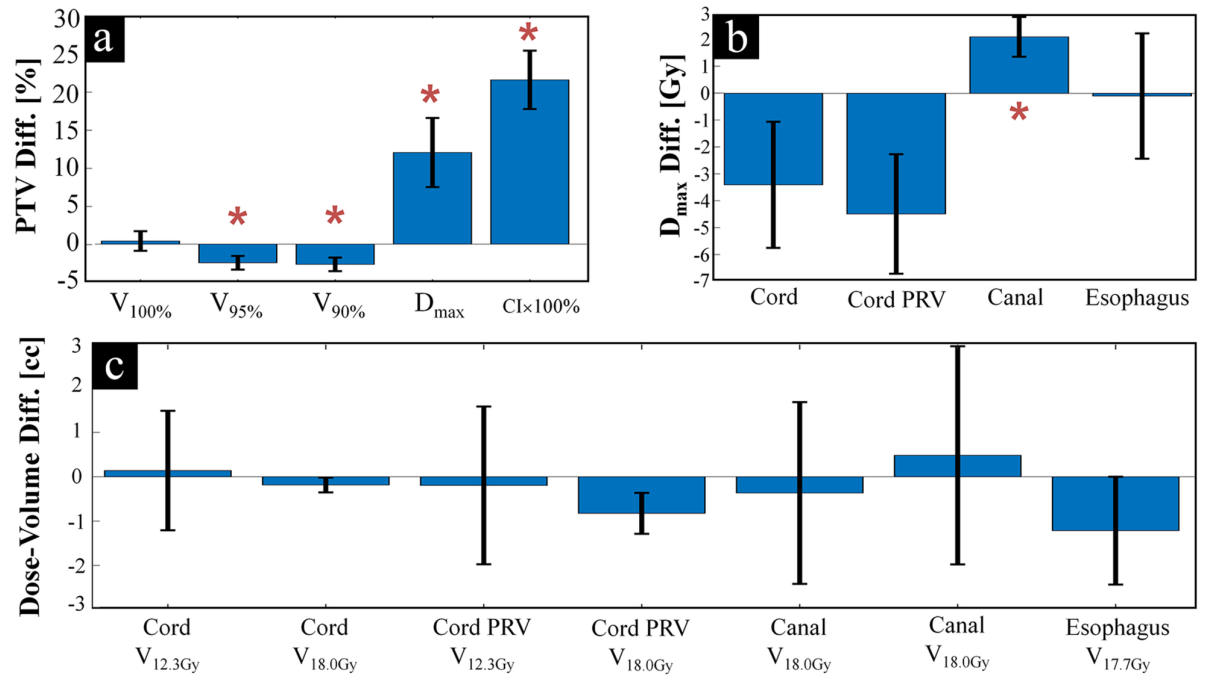

\section{FIGURE 3: Quantitative Dosimetric Comparison}

Quantitative dosimetric comparison of planning target volume (PTV) parameters displayed in (a), Organ-atrisk/Planning-organ-at-risk-volume (OAR/PRV) max dose parameters are displayed in (b), and OAR/PRV dose-volume parameters are displayed in (c). Asterisks indicate statistically significant $(p<0.05)$ differences. Differences are magnetic resonance guided-linac (MR-Linac) minus volumetric modulated arc therapy (VMAT) values. Error bars show standard deviation of this difference.

\section{Plan deliverability}

The planning comparison in Figure 2 and Figure 3 demonstrate a theoretical capability of the ViewRay MRLinac to produce spine SBRT plans meeting clinical goals for various targets. To validate true deliverability, phantom measurements with various dosimeters assessed fidelity to planned dose distributions. 


\section{Cureus}

One aspect of deliverability is treatment time. Planning strategies avoiding excessive delivery times on the MR-linac are necessary. The number of segments during optimization was restricted and the IMRT "efficiency" parameter within the TPS was increased to encourage larger beam segments. The MR-linac plans consisted of the same nine gantry angles $\left(40^{\circ}\right.$ separation starting at $\left.0^{\circ}\right)$. The number of segments per IMRT beam were $9.21 \pm 0.48$ (range: 2 -21). The planning system calculated beam on time was $12.75 \pm 1.27$ minutes (range: 8.85-17.92 minutes). Including gantry and MLC motion, the MR-linac treatment times were $14.39 \pm 1.26$ minutes (range: $10.08-18.96$ minutes), compared to the clinical VMAT treatment times of $9.57 \pm 1.19$ minutes $(\mathrm{p}=0.0202)$. The MR-linac treatment times are significantly longer, but are still clinically reasonable.

Table 2 summarizes the phantom measurement results for cases $\# 7$ and $\# 8$ (representing the spectrum of potential PTV geometries, see Table 1 and Figure 1 ). Gamma passing rates for the diode array phantom and the film measurements are excellent with $3 \% / 3 \mathrm{~mm}$ and acceptable with $2 \% / 2 \mathrm{~mm}$. Ion chamber measurements at a single point shows clinically acceptable fidelity ( $<3 \%$ difference).

\begin{tabular}{|l|llll|l|}
\hline & ArcCHECK & & Film & & Ion Chamber (Dose Difference) \\
\hline Gamma Criteria: & $3 \% / 3 \mathrm{~mm}$ & $2 \% / 2 \mathrm{~mm}$ & $3 \% / 3 \mathrm{~mm}$ & $2 \% / 2 \mathrm{~mm}$ & \\
Case \#7 & $100.0 \%$ & $99.3 \%$ & $98.0 \%$ & $92.7 \%$ & $+0.7 \%$ \\
Case \#8 & $99.4 \%$ & $95.3 \%$ & $96.4 \%$ & $89.3 \%$ & $+2.5 \%$ \\
\hline
\end{tabular}

\section{TABLE 2: Phantom Measurement Results}

Summary of results comparing measured to planned doses for cases \#7 and \#8. Gamma passing rates for both ArcCHECK diode and radiochromic film measurements using various gamma criteria are shown as well as a relative dose difference between ion chamber measured and planned dose.

Film analysis is shown in Figure 4 and Figure 5 for cases \#7 and \#8, respectively. In Figure 5, the low-dose region for spinal cord sparing can be seen inside of the high-dose region used to treat the disease.

Orthogonal dose profiles in Figure 4 and Figure 5 show closely matching spatial doses, which is particularly impressive in the high-gradient regions between the target and spinal cord. Regions failing gamma analysis for $3 \% / 3 \mathrm{~mm}$ and $2 \% / 2 \mathrm{~mm}$ criteria are shown in red in Figures $4 e-4 f$ and Figures $5 e-5 f$. Figure $4 g$ and Figure $5 \mathrm{~g}$ shows similar dose profiles from diode array measurements.
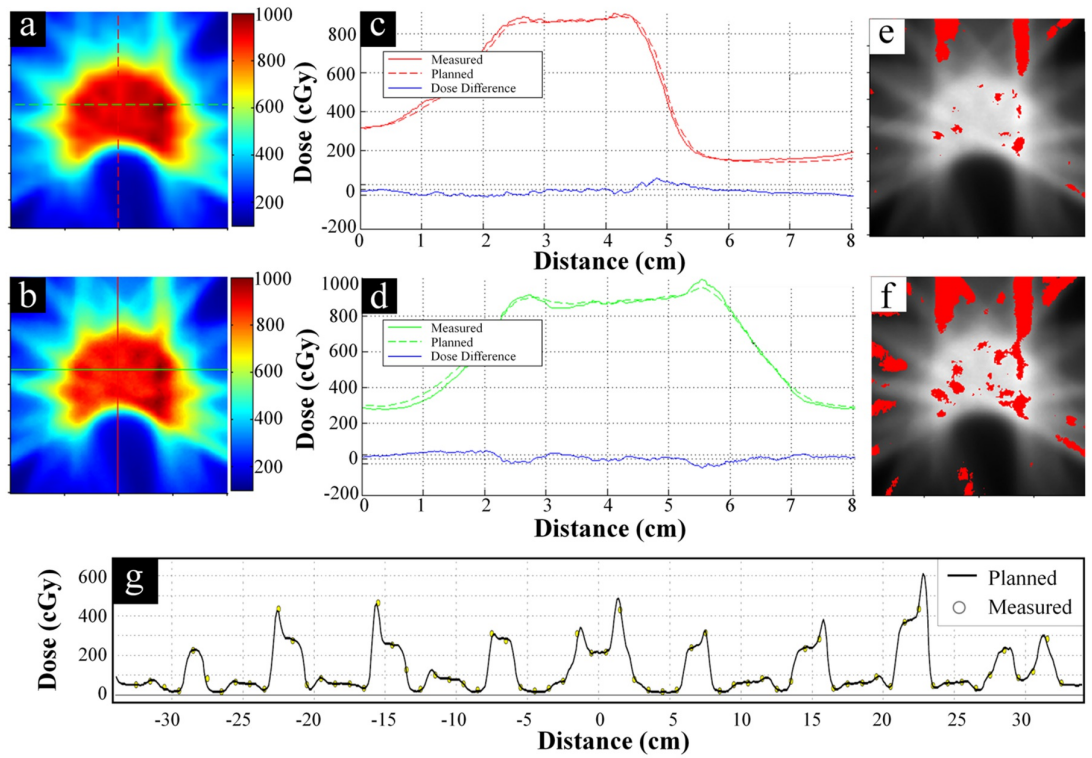

\section{FIGURE 4: Case \#7 - Simple PTV Geometry}

Spatial dosimetry results for case \#7 representing simple planning target volume (PTV) geometry. (a) Planned and (b) measured planar dose distributions. (c) Vertical and (d) horizontal dose profiles along the red and green lines, respectively, shown in (a) and (b). Solid lines are measured profiles, dotted lines are planned profiles, and the blue line in (c) and (d) shows the difference between the two. Gamma analysis results when using either $3 \% / 3 \mathrm{~mm}$ or $2 \% / 2 \mathrm{~mm}$ criteria are shown in (e) and (f), respectively. Measured dose distribution is shown in grayscale and red points indicate a gamma value $>1$ (i.e., failing points). (g) Representative dose profiles showing ArcCHECK diode measured (circles) versus planned (solid line) dose distributions. Yellow 

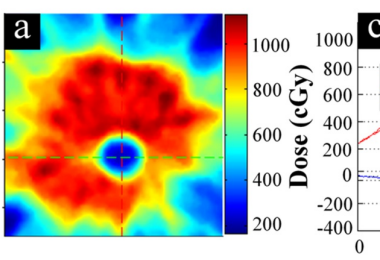

\section{d}

b
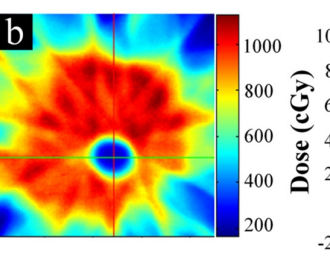

1000 d
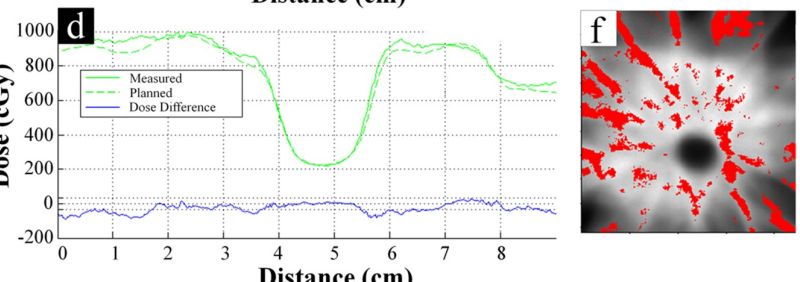

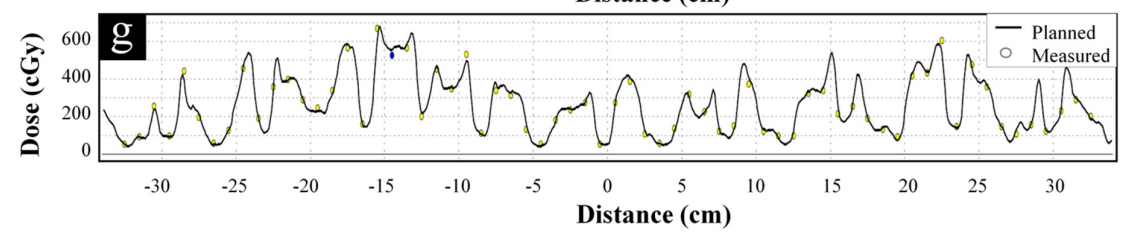

\section{FIGURE 5: Case \#8 - Complex PTV Geometry}

Spatial dosimetry results for case \#8 representing a complex planning target volume (PTV) geometry. (a) Planned and (b) measured planar dose distributions. (c) Vertical and (d) horizontal dose profiles along the red and green lines, respectively, shown in (a) and (b). Solid lines are measured profiles, dotted lines are planned profiles, and the blue line in (c) and (d) shows the difference between the two. Gamma analysis results when using either $3 \% / 3 \mathrm{~mm}$ or $2 \% / 2 \mathrm{~mm}$ criteria are shown in (e) and (f), respectively. Measured dose distribution is shown in grayscale and red points indicate a gamma value $>1$ (i.e., failing points). (g) Representative dose profiles showing ArcCHECK diode measured (circles) versus planned (solid line) dose distributions. Yellow filled circles pass gamma analysis and blue/red filled circles fail with lower/higher measured dose.

\section{Discussion}

Recent work found the MR-linac to be capable of cranial stereotactic radiosurgery (SRS) [18]. Extracranial or spinal SRS/SBRT utilizes similar margins $(\sim 1 \mathrm{~mm})$ and requires similar precision/accuracy. This is facilitated by improvements between the ${ }^{60} \mathrm{Co}$ and MR-linac iterations including: 6MV flattening filter free (FFF) beam (decreased penumbra, lower surface dose, higher penetration, and non-decaying $600 \mathrm{cGy} / \mathrm{min}$ dose rate), a 138 leaf double-stacked double-focused MLC system (effectively projecting to $0.415 \mathrm{~cm}$ rather than $1.05 \mathrm{~cm}$ at isocenter, enabling $0.2 \mathrm{~cm} \mathrm{x} 0.4 \mathrm{~cm}$ field sizes and decreasing interleaf leakage) $[15,18]$. The relatively low magnetic field $(0.35 \mathrm{~T})$ decreases distortion and provides imaging accuracy better than $1 \mathrm{~mm}$ with registration accuracy on the order of a single voxel $(\sim 1.5 \mathrm{~mm})[22]$.

Preliminary studies show that the ViewRay MRIdian linac can visualize the cord/cauda versus surrounding thecal sac [16]. While spine SBRT patients have been successfully and safely treated with current nonMRgRT approaches, most rely on bony anatomy as a surrogate for the true OAR (cord). PRV expansion partly accounts for cord motion independent of surrounding vertebra. However, registering images capturing intrafraction motion to initial planning images based on bony anatomy or the cord itself resulted in average setup differences of $0.9 \mathrm{~mm}$ (up to $1.8 \mathrm{~mm}$ ) [16]. This suggests that we can improve spine SBRT by registering directly to the spinal cord. With no clear time trends to such motion, continuous/real-time image guidance, as provided by MR-linac may be necessary [16]. A system that can visualize the dose-limiting cord in real-time potentially enables cord-based gating. However, this may lengthen treatment times.

Different general approaches to online adaptive RT are discussed in the literature [7,23-25]. The work presented herein argues for spine SBRT implementation on the MRIdian MR-linac platform, but the proper adaptive approaches must be determined. Online plan reoptimization for spine SBRT must be investigated as has been done for other sites [24-26]. More accurate image guidance, gating, and/or adaptive RT for spine SBRT on the MR-linac may enable reduced margins and dose escalation for improved efficacy.

Compared to conventional linac VMAT plans, increased heterogeneity (greater Dmax) and inferior conformality (particularly for intermediate/lower isodoses) found in this work are consistent with previous studies [12]. It is important to emphasize that the heterogeneity, while higher for the MR-linac plans, was 
found to be clinically acceptable for treatments of this nature, as it facilitates the necessary steep dose fall off to spare the nearby spinal cord. For this work, nine equally-spaced, fixed IMRT fields were used. Prior studies found no significant improvements with greater than nine beams [27]. While presented results show dosimetric capabilities needed for spine SBRT, approaches may be improved (e.g., plan specific beam angles, off-axis treatments approximating non-coplanar beams, alternative optimization approaches, additional delivery efficiency considerations, etc.). Clinical VMAT plans had more freedom to utilize variable numbers of arcs (2-4), collimator angles, etc., based on target geometry and relationship to nearby OARs. Target and OAR contours were kept consistent between clinical VMAT and MR-linac plans. Other planning studies have incorporated assumed benefits of MRgRT on PTV/PRV margins and utilized smaller targets in the MRgRT plans, providing a dosimetric advantage [11,13-14]. In fact, the previous study exploring spine SBRT for the Cobalt system used this approach (VMAT: $1 \mathrm{~mm}$ cord PRV vs. ViewRay: cord PRV = true cord). Regardless, while the cord was well-visualized, dosimetry was inadequate [13]. The single fraction prescription of $18 \mathrm{~Gy}$ was decreased to $12.6 \mathrm{~Gy}$ on average to respect cord tolerance (versus $17.5 \mathrm{~Gy}$ for VMAT, which used an additional $1 \mathrm{~mm}$ margin for cord PRV). Average beam-on time (48.82 minutes) was clinically unacceptable [13]. With the MR-linac, target coverage is comparable to VMAT and clinically acceptable in all cases. While beam on time is longer than for VMAT, beam-on times with the 6MV FFF beam are acceptable $(12.75 \pm 1.27$ minutes), and related intrafraction motion issues arising from longer treatment times could potentially be offset by leveraging the OAR-based real-time tracking capabilities of the MR-Linac. Note, conventional linac plans used a 6MV beam (max dose rate of $600 \mathrm{MU} / \mathrm{min}$ ), but if $6 \mathrm{MV}$ FFF (max dose rate of $1400 \mathrm{MU} / \mathrm{min}$ ) were utilized, average treatment time $(9.57 \pm 1.19 \mathrm{~min})$ would decrease. It should be noted that the treatment planner was different for the MR-Linac plans and the clinical VMAT plans and that the MR-Linac planner was not necessarily blinded to the dosimetry of the baseline clinical VMAT plans.

Many spine SBRT patients require orthopedic hardware for spinal stabilization. While MR-compatible, it is unclear how susceptibility artifacts may affect MRgRT spine SBRT. However, a study investigating the MRIdian for high-dose rate (HDR) brachytherapy planning found decreased artifacts from applicators/instruments in $0.35 \mathrm{~T}$ versus higher-field diagnostic MRI [28]. MRI provides both functional and anatomical information. Diffusion-weighted imaging has already been demonstrated on the MRIdian [29]. As pulse-sequence options continue to expand, so will interesting clinical applications.

\section{Conclusions}

This work demonstrates the feasibility of spine SBRT with fixed-field IMRT on the ViewRay MR-linac. Compared to clinical VMAT plans on a conventional linac, MR-linac plans had increased heterogeneity, lower conformality and longer beam-on. However, MR-linac plans were dosimetrically clinically acceptable and, while not statistically significant, tended to have improved cord sparing. Phantom measurements of delivered doses with an ion chamber, film, and diode array show good fidelity with planned doses, particularly in the regions between target and cord with large dose gradients, ensuring that the dose distributions are deliverable. MRgRT Spine SBRT is an application that may allow direct visualization of the dose-limiting spinal cord in real-time during treatment for reduced margins, dose escalation, gating, and/or adaptive radiotherapy.

\section{Additional Information \\ Disclosures}

Human subjects: Consent was obtained by all participants in this study. Animal subjects: All authors have confirmed that this study did not involve animal subjects or tissue. Conflicts of interest: In compliance with the ICMJE uniform disclosure form, all authors declare the following: Payment/services info: All authors have declared that no financial support was received from any organization for the submitted work. Financial relationships: All authors have declared that they have no financial relationships at present or within the previous three years with any organizations that might have an interest in the submitted work. Other relationships: All authors have declared that there are no other relationships or activities that could appear to have influenced the submitted work.

\section{Acknowledgements}

The authors would like to acknowledge Anett Young for her hard work and assistance in facilitating this multi-institutional collaborative work. This research did not receive any specific grant from funding agencies in the public, commercial, or not-for-profit sectors.

\section{References}

1. Redmond KJ, Robertson S, Lo SS, et al.: Consensus contouring guidelines for postoperative stereotactic body radiation therapy for metastatic solid tumor malignancies to the spine. Int J Radiat Oncol Biol Phys. 2017, 97:64-74. 10.1016/j.ijrobp.2016.09.014

2. Cox BW, Spratt DE, Lovelock M, et al.: International spine radiosurgery consortium consensus guidelines for target volume definition in spinal stereotactic radiosurgery. Int J Radiat Oncol Biol Phys. 2012, 83:e597e605. 10.1016/j.ijrobp.2012.03.009

3. Hamilton AJ, Lulu BA, Fosmire H, Stea B, Cassady JR: Preliminary clinical experience with linear accelerator-based spinal stereotactic radiosurgery. Neurosurgery. 1995, 36:311-319. 10.1227/00006123- 
199502000-00010

4. Takacs I, Hamilton AJ: Extracranial stereotactic radiosurgery: applications for the spine and beyond . Neurosurg Clin N Am. 1999, 10:257-270. https://doi.org/10.1016/S1042-3680(18)30192-X

5. Ryu SI, Chang SD, Kim DH, Murphy MJ, Le QT, Martin DP, Adler JR Jr.: Image-guided hypo-fractionated stereotactic radiosurgery to spinal lesions. Neurosurgery. 2001, 49:838-846. 10.1097/00006123-20011000000011

6. Gerszten PC, Ozhasoglu C, Burton SA, Vogel WJ, Atkins BA, Kalnicki S, Welch WC: CyberKnife frameless stereotactic radiosurgery for spinal lesions: clinical experience in 125 cases. Neurosurgery. 2004, 55:89-98. https://doi.org/10.1227/01.neu.0000440704.61013.34

7. Mutic S, Dempsey JF: The ViewRay system: magnetic resonance-guided and controlled radiotherapy. Semin Radiat Oncol. 2014, 24:196-199. 10.1016/j.semradonc.2014.02.008

8. Lagendijk JJ, Raaymakers BW, van Vulpen M: The magnetic resonance imaging-linac system. Semin Radiat Oncol. 2014, 24:207-209. 10.1016/j.semradonc.2014.02.009

9. Wee CW, An HJ, Kang HC, Kim HJ, Wu HG: Variability of gross tumor volume delineation for stereotactic body radiotherapy of the lung with tri-(60)Co magnetic resonance image-guided radiotherapy system (ViewRay): a comparative study with magnetic resonance- and computed tomography-based target delineation. Technol Cancer Res Treat. 2018, 17:10.1177/1533033818787383

10. Noel CE, Parikh PJ, Spencer CR, Green OL, Hu Y, Mutic S, Olsen JR: Comparison of onboard low-field magnetic resonance imaging versus onboard computed tomography for anatomy visualization in radiotherapy. Acta Oncol. 2015, 54:1474-1482. 10.3109/0284186X.2015.1062541

11. Park JM, Park SY, Kim HJ, Wu HG, Carlson J, Kim JI: A comparative planning study for lung SABR between tri-Co-60 magnetic resonance image guided radiation therapy system and volumetric modulated arc therapy. Radiother Oncol. 2016, 120:279-285. 10.1016/j.radonc.2016.06.013

12. Wooten HO, Green O, Yang M, et al.: Quality of intensity modulated radiation therapy treatment plans using a 60Co magnetic resonance image guidance radiation therapy system. Int J Radiat Oncol Biol Phys. 2015, 92:771-778. 10.1016/j.ijrobp.2015.02.057

13. Choi CH, Park SY, Kim JI, Kim JH, Kim K, Carlson J, Park JM: Quality of tri-Co-60 MR-IGRT treatment plans in comparison with VMAT treatment plans for spine SABR. Br J Radiol. 2017, 90:1070. 10.1259/bjr.20160652

14. Park JM, Park SY, Kim JI, Kang HC, Choi CH: A comparison of treatment plan quality between Tri-Co-60 intensity modulated radiation therapy and volumetric modulated arc therapy for cervical cancer. Phys Med. 2017, 40:11-16. 10.1016/j.ejmp.2017.06.018

15. Kluter S: Technical design and concept of a 0.35 T MR-Linac . Clin Transl Radiat Oncol. 2019, 18:98-101. 10.1016/j.ctro.2019.04.007

16. Robinson C, Bradley J, Victoria J, Dempsey J, Mutic S, Kashani R: Comparison of spinal cord motion versus vertebral body motion during magnetic resonance imaging-guided radiation therapy (MR-IGRT). Int J Radiat Oncol Biol Phys. 2014, 90:301-302. https://doi.org/10.1016/j.ijrobp.2014.05.1012

17. Benedict SH, Yenice KM, Followill D, et al.: Stereotactic body radiation therapy: the report of AAPM Task Group 101. Med Phys. 2010, 37:4078-4101. 10.1118/1.3438081

18. Wen N, Kim J, Doemer A, et al.: Evaluation of a magnetic resonance guided linear accelerator for stereotactic radiosurgery treatment. Radiother Oncol. 2018, 127:460-466. 10.1016/j.radonc.2018.04.034

19. Wooten HO, Rodriguez V, Green O, et al.: Benchmark IMRT evaluation of a Co-60 MRI-guided radiation therapy system. Radiother Oncol. 2015, 114:402-405. 10.1016/j.radonc.2015.01.015

20. Li HH, Rodriguez VL, Green OL, et al.: Patient-specific quality assurance for the delivery of (60)Co intensity modulated radiation therapy subject to a 0.35-T lateral magnetic field. Int J Radiat Oncol Biol Phys. 2015, 91:65-72. 10.1016/j.ijrobp.2014.09.008

21. O'Brien DJ, Roberts DA, Ibbott GS, Sawakuchi GO: Reference dosimetry in magnetic fields: formalism and ionization chamber correction factors. Med Phys. 2016, 43:4915. 10.1118/1.4959785

22. Ginn JS, Agazaryan N, Cao M, et al.: Characterization of spatial distortion in a 0.35 T MRI-guided radiotherapy system. Phys Med Biol. 2017, 62:4525-4540. 10.1088/1361-6560/aa6e1a

23. Fischer-Valuck BW, Henke L, Green O, et al.: Two-and-a-half-year clinical experience with the world's first magnetic resonance image guided radiation therapy system. Adv Radiat Oncol. 2017, 2:485-493. 10.1016/j.adro.2017.05.006

24. Lamb J, Cao M, Kishan A, et al.: Online adaptive radiation therapy: implementation of a new process of care . Cureus. 2017, 9:e1618. 10.7759/cureus.1618

25. Acharya S, Fischer-Valuck BW, Kashani R, et al.: Online magnetic resonance image guided adaptive radiation therapy: first clinical applications. Int J Radiat Oncol Biol Phys. 2016, 94:394-403. 10.1016/j.ijrobp.2015.10.015

26. Henke L, Kashani R, Robinson C, et al.: Phase I trial of stereotactic MR-guided online adaptive radiation therapy (SMART) for the treatment of oligometastatic or unresectable primary malignancies of the abdomen. Radiother Oncol. 2018, 126:519-526. 10.1016/j.radonc.2017.11.032

27. Fox C, Romeijn HE, Lynch B, Men C, Aleman DM, Dempsey JF: Comparative analysis of 60Co intensitymodulated radiation therapy. Phys Med Biol. 2008, 53:3175-3188. 10.1088/0031-9155/53/12/007

28. Ko HC, Huang JY, Miller JR, et al.: Novel use of ViewRay MRI guidance for high-dose-rate brachytherapy in the treatment of cervical cancer. Brachytherapy. 2018, 17:680-688. 10.1016/j.brachy.2018.04.005

29. Yang Y, Cao M, Sheng K, et al.: Longitudinal diffusion MRI for treatment response assessment: preliminary experience using an MRI-guided tri-cobalt 60 radiotherapy system. Med Phys. 2016, 43:1369-1373. $10.1118 / 1.4942381$ 